\title{
Automated system for data acquisition and monitoring
}

\author{
Sorin Borza ${ }^{1, *}$, and Iunia Cristina Borza ${ }^{2}$ \\ ${ }^{1}$ University Lucian Blaga of Sibiu, Romania \\ ${ }^{2}$ Microsoft Iberica, Paseo del Club Deportivo, 1Centro Empresarial La Finca - Edificio 1 \\ 28223 Pozuelo de Alarcón (Madrid), Spain
}

\begin{abstract}
The Environmental management has become, with the development of human society a very important issue. There have been multiple systems that automatically monitors the environment. In this paper we propose a system that integrates GIS software and data acquisition software. In addition the proposed system implements new AHP multicriteria method that can get an answer online on each pollutant influence on limited geographical area in which the monitors. Factors pollutants of limited geographical areas are taken automatically by specific sensors through acquisition board. Labview software, with virtual instrument created by transferring them into a database Access. Access database they are taken up by software Geomedia Professional and processed using multi-criteria method AHP, so that at any moment, their influence on the environment and classify these influences, can be plotted on the screen monitoring system. The system allows, the automatic collection of data, the memorization and the generation of GIS elements. The research presented in this paper were aimed at implementing multicriteria methods in GIS software.
\end{abstract}

\section{Introduction}

With the development of human society has become obvious that It affects the environment and human activity is in turn influenced by to it. Passing through various stages of organization, quality protection environment and new integrated approach to environmental management become a vital necessity to ensure the prosperity of society and sustainable development.

Environmental management has become an essential part for any type of development regardless of scale that may occur environmental impacts. Environment monitoring system is an integrated system that continues to supervise the state of the environment and provides data on all of its structural components (air, water, soil, biocoenosis). The data acquired are processed by specific methods, and the information thus obtained is used for the final assessment of the environmental impact, for warning and alarm and control of the environment.

\footnotetext{
* Corresponding author: sorin.borza@ulbsibiu.ro
} 
The main purpose of an environmental monitoring system regardless of scale covered or the number of components that includes is to provide an objective view as more realistic environment for the adoption of corrective measures control pollution and recovery [1].

Integrated monitoring aimed at achieving true-overall environmental quality of the stage at a time and historical trend two basic components - the biotic and abiotic environment - in their interconnection. From passive phase highlight the state of quality monitoring is currently an active intervention, control and evaluation of the effectiveness of regulatory measures, strategies profile and generation needs to promote clean technologies [1].

The integrated monitoring ensures database for both depth research (understanding, explanation and evaluation quantified the various ecosystems in terms of structure, processes and mechanisms operating within the hierarchy of ecological systems), and assessing the impact of actions undertaken to maintain the integrity of ecological systems [2].

Monitoring can be done for various purposes, including to establish "baselines, trends and cumulative effects" on the environment [3], to improve process modeling environment, to educate the public on environmental conditions, to support the decision-making environment for compliance with environmental regulations, to assess effects anthropogenic influences on the environment, or perform an inventory of natural resources [3]. Local level monitoring refers mainly to environmental observations near sources that supposedly can cause impacts. Apart from this restricted from another point of view the local concerns and national monitoring systems, relative to the overall system.

Environmental monitoring system works similarly to a double hierarchy system in business management and information system.

It is characterized by a functional interdependence between its five components, namely:

- supervision;

- evaluation of the actual state of the environment;

- prognostication state environmental;

- assessment of prognosis;

- adjust the quality of the environment.

Key indicators of the European Environment Agency (EEA) supports the monitoring and implementation of the priority objectives of EU environmental policy, is regularly updated [4]. They are bound by obligations of European Union resulting from the signing of agreements, treaties or international protocols targets assumed under various directives (eg Water Framework Directive, the Waste Framework Directive, the Habitats Directive, Birds Directive) or attempts of solving direct problems incidence in the quality of housing at community. Exist the 37 key indicators monitored by the European Environment Agency (Core Set indicators - CSI) [5].

In Automatic Environmental Monitoring System were used IEPE sensors and acquisition board, NI 9234 NI Mseries. The Drager Pac III apparatus has been used for the measurement of the monoxide carbon. For the sound measurements EXTECH 407780 was used.

The automatic taking over and the analysis of the data using the AHP method (Analytic Hierarchy Process) and of generating the element's classes necessary to elaborate the intelligent maps consists of hardware components: sensors of the acquisition board, apparatus used for measuring the polluting factors and also, of software elements, like: LabVIEW, Geomedia Professional, Access Database, presented in figure1. 


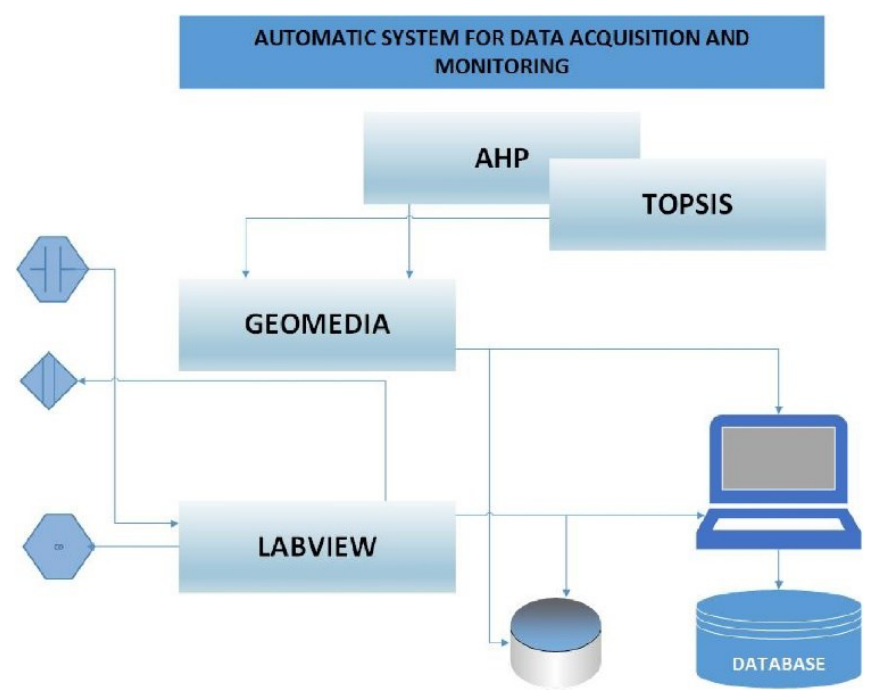

Fig. 1. The system for Acquisition and Monitoring Data

The MCDM methods are frequently used to solve real-world problems with multiple attributes. Recently, various methods have been developed to support decision making in various fields. Multi-criteria decision making (MCDM) is a well-known branch that deals with problems making decisions based on a number of criteria.

\section{The Object Oriented Programming in LABVIEW}

In object oriented programming with LabVIEW, the concept of "Dataflow" is extented [6]. In LVOOP, a class consists merely of a user defined data type together with methods that can be applied to values of that data type. Once could say that object oriented programming in Labview allows the developer to create object oriented wires.

As in other object oriented languages, a derived class may overload an abstract method of its base class. However, the override method must have exactly the same input and output parameters as the respective method of the parent class. The concept of dataflow requires objects to flow through their "class wires" from source to drain [7].

The fundamental differences between LVOOP and conventional object oriented languages prevent a straightforward implementation of design patterns that are based on the idea of objects as entities. However, many of those design patterns are useful for designing control systems. LabVIEW contains several built-in reference-based components, such as File I/O, TCP/IP Networking and ActiveX Automation. Using the LVOOP approach we can take advantage of the benefits of object-based design and development. Adding the class and object concepts from other object-oriented languages to LabVIEW, you can build your own components. By implementing classes, you can take advantage of the design and development practices used by developers who use standard OO languages. You will also be able to use standard $\mathrm{OO}$ analysis and design tools, such as Rational Rose and Microsoft Visual Modeler and apply principles described in literature about OO design and development.

The component-based approach to developing application augments the traditional dataflow programming used in LabVIEW applications. You will develop large applications by identifying and designing components that implement distinct parts of the functionality of the application [8]. 


\section{The MCDM METHODS}

The Multicriterial evaluation is used in cases where there are several alternatives, variations, locations or processes that have to be assessed by their total environmental load or quality. The common result of multicriterial evaluation methods is dimensionless number that indicates the degree of environmental load of alternatives that are valued. In addition to indicators that represent the environmental impact it is possible to include indicators that have economic, social, and technological character.

\subsection{The AHP method}

AHP (Analytical Hierarchy Process) is a structured technique for solving complex decision developed by Thomas L. Saaty [9]. This technique allows the decision maker to take the most appropriate decisions for him by failing necessarily the right decision.

This method is based on splitting the problem into simpler subproblems ranking / ordering that can be analyzed independently of each other. Once constructed hierarchy, decision maker evaluates its elements by comparing their successive pairs. These comparisons reflect the main advantage of the approach, namely that it necessarily requires the use of objective and clearly defined, allowing comparisons on the basis of purely subjective and ambiguous. All of these orderings are converted into numerical values, which will then allow the aggregation results obtained in such a way that the solution to the solution to the original problem.

An important component of the AHP method is a mathematical model by which priorities of elements are calculated (weighted), for elements that are on the same level hierarchical structure. AHP was successfully used in environmental impact assessment for determining of weights for impact categories [10]. AHP was used for verification of results gained by quantification of environmental aspects and impacts [11]. Summary of AHP method consists of converting subjective assessments to the relative importance of the criteria scores and weights. The AHP method was developed by Saaty [12] and is one of the most used methods MCDM.

In the method AHP each criterion is compared with the other subjective criteria considered by method. This results are compared in pairs, resulting are in scores and weights. For each criterion is taken into account Table 1:

Table 1. The Saaty table

\begin{tabular}{|l|c|}
\hline \multicolumn{1}{|c|}{ Definition } & Importance Intensity \\
\hline Equally important. Compare criteria with itself & $\mathbf{3}$ \\
\hline $\begin{array}{l}\text { The Criterion is more important than the criterion } \\
\text { they compare }\end{array}$ & $\mathbf{5}$ \\
\hline $\begin{array}{l}\text { The Criterion is strongly more important than the } \\
\text { criterion they compare }\end{array}$ & $\mathbf{7}$ \\
\hline $\begin{array}{l}\text { The Criterion is very strongly more important than } \\
\text { the criterion they compare }\end{array}$ & $\mathbf{9}$ \\
\hline $\begin{array}{l}\text { The Criterion is extremely more important than the } \\
\text { criterion they compare }\end{array}$ & \\
\hline Intermediate values are allowed: $2,4,6,8$ & \\
\hline
\end{tabular}

The intermediate values are allowed to shade difference between the two criteria. The total number of evaluations will be:

$$
\frac{1}{2} \times n \times(n-1)
$$


Using this method the following four steps:

Step 1. Defining decision criteria form a hierarchy of objectives. On the upper level is the objective of the problem, the decision criteria are intermediate levels and the lower level, alternatives / variants decision. A priority hierarchy is a system and organization of concepts in which each element, with the exception of the upper level, is subject one or more elements of the hierarchy. AHP hierarchy involves placing the higher purpose / primary objective, and then placing the decision criteria to intermediate levels, possibly broken down into subcriteria, if necessary. The lowest are found alternatives decision to be ordered by preference decision maker / decision makers.

This is essentially an alternative multi-criteria decision modeling problem under deterministic. A graphical representation of this hierarchy is presented in figure 2.

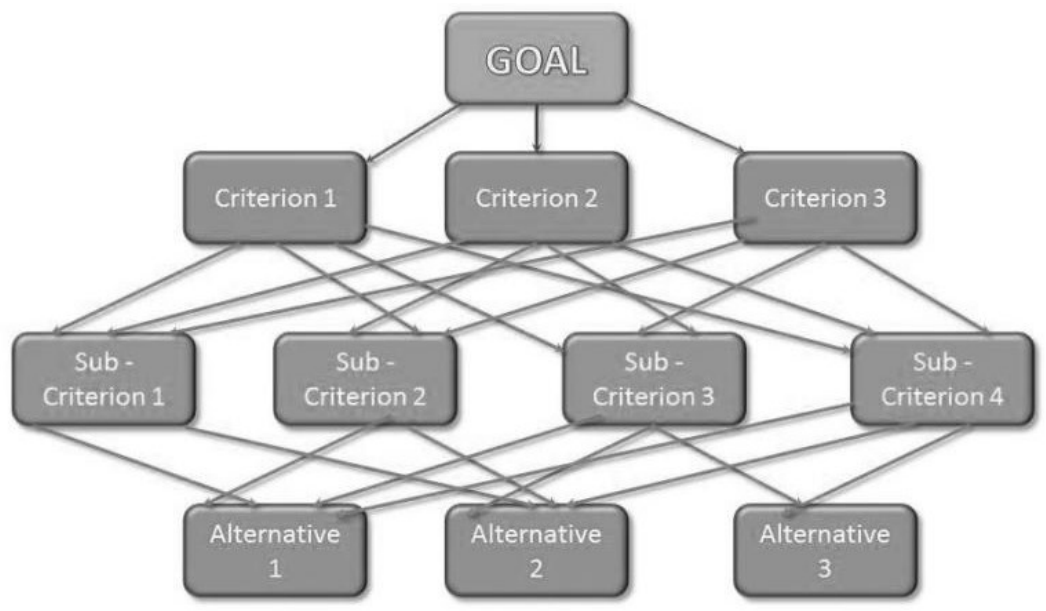

Fig. 2 General hierarchical model in AHP

Originally established hierarchy can be modified during the course of decision-making by identifying new nodes.

Step 2. Weight criteria, sub-criteria and alternatives according to their importance for higher level correspondent. To achieve this ordering using simple comparisons.

At this stage, the priorities established for the hierarchy nodes. The priorities are numerical values in the range $[0,1]$ and represent the weights associated with nodes of said node relative to any group.

By definition, the share associated upper level / target is 1.00 . For each level in the hierarchy relative weights sum will be 1.00 .

The significance of these contributions is to show how to calculate them. A node with 0.20 weight will be twice as important than the 0.10 significance node. This way of quantifying the preferences that permits not only the ordering of preferences of a decision maker, but also quantify the intensity of this preference. Two concepts are closely related to the hierarchy of local priorities and global priorities. Local priorities are above and independent of the hierarchy that is node, while global priorities are obtained by weighting local priorities all levels weights directly above the level considered. Thus, the overall sum of the weights of the nodes-be is always equal to the overall share of the parent node. Although priorities goal, decision criteria and alternatives are closely interrelated, the AHP method is to take them as independent values [12].

These values of weights are then modified to obtain a decision matrix. These modifications are made by successive comparisons of the elements of the problem, two by two.

It compares each of the same hierarchical level elements which have the same parent node using table 1 . 
Step 3 Local priorities (weights) of criteria, sub-criteria and alternatives at same hierarchical structure level are calculated through appropriate mathematical model and afterwards they are synthesized in total priorities of alternatives.

Step 4 Implementation of the sensitivity analysis for final decisions.

$$
A=\left[\begin{array}{cccc}
1 & a_{12} & \cdots & a_{1 j} \\
a_{21} & 1 & \cdots & a_{2 j} \\
\vdots & \vdots & \ddots & \vdots \\
a_{i 1} & a_{i 2} & \cdots & a_{i j}
\end{array}\right]
$$

\section{Fig. 3. Matrix A}

The matrix $A$, figure 3, has special features (all of it's rows are proportional to the first row, and they are all positive and $a i j=1 /$ aji is true) and because of that only one of it's eigenvalue differs from 0 and is equal to $n$.

If the matrix $A$ contains inconsistent estimates (in practical examples almost always), weight vector $w$ can be obtained by solving the equation $(A-\lambda \max I) w=0$ with prerequisite that $\Sigma$ wi $=1$, where $\lambda \max$ is the largest eigenvalue in matrix $A$. Because of matrix $A$ properties $\lambda$ max $\geq n$, the difference $\lambda \max -n$ is used in measuring timations consistency. With consistency index

$$
C I=(\lambda \max -n) /(n-1)
$$

After consistency index, consistency can be calculated:

$$
C R=C I / R I
$$

In the method developed by Saaty [9], the calculation of the weights is based on a relatively complex mathematical apparatus, using matrix algebra tools. The results are values associated to eigenvector of maximum eigenvalue matrix. The calculations are quite complex, so it is necessary to use a dedicated program.

But in practice, we provide a simple method of calculation, which gives the same result with two decimal places:

- Calculate the geometric mean of each row of the matrix.

- It calculates the sum of the geometric mean.

- Normalized geometric mean.

\section{Results and discussions}

In the automatic system, measurements can be taken automatically by sensors connected to computer through data acquisition boards or measurements can be made directly with the apparatus dedicated to polluant factors. The data can be retrieved by means of the following IEPE sensors: SM 70 ozone fixed sensor, dust sensor g2yp1010 for dust particle, MQ-5 sensor for detect nitrogen oxide and NI MyRIO-1900 acquisition board.

This board allows connection to LABVIEW software environment and having a specific driver for connection. Environmental pollution data, figure 5, are captured by means of sensors and acquisition board placed in the Access database attached to the virtual machine. The MCDM AHP method, is implemented in Labview virtual machine and provides specific data for each measurement point. 


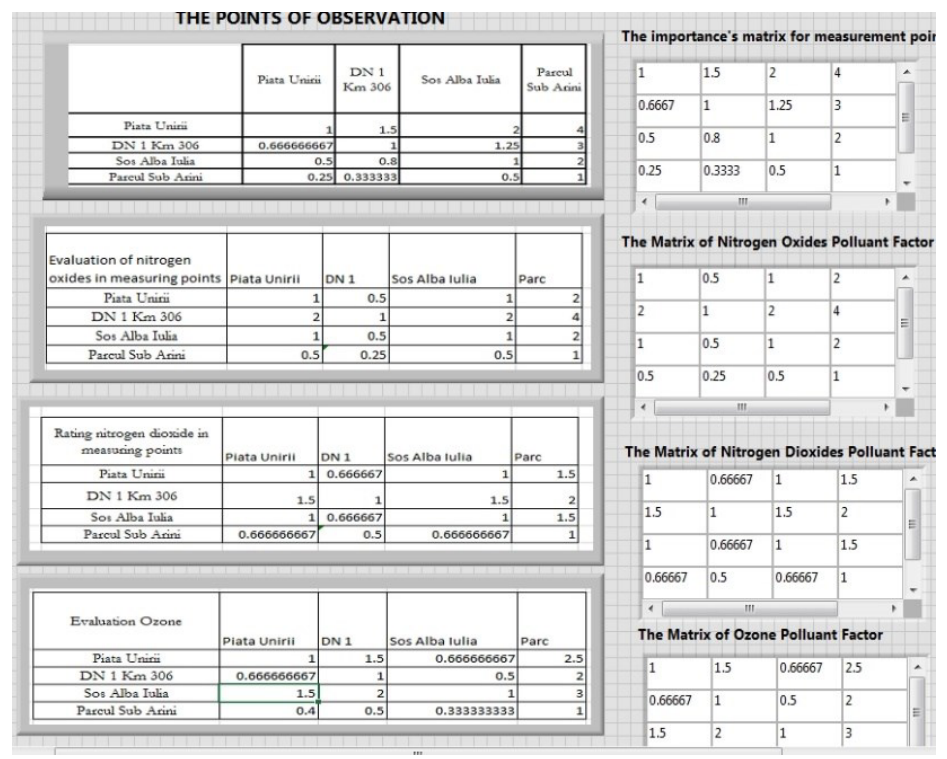

Fig. 5. The Input data for Virtual Instrument

The data is taken in Labview software, where was created a virtual instrument, whose panel is shown in Figure 6.

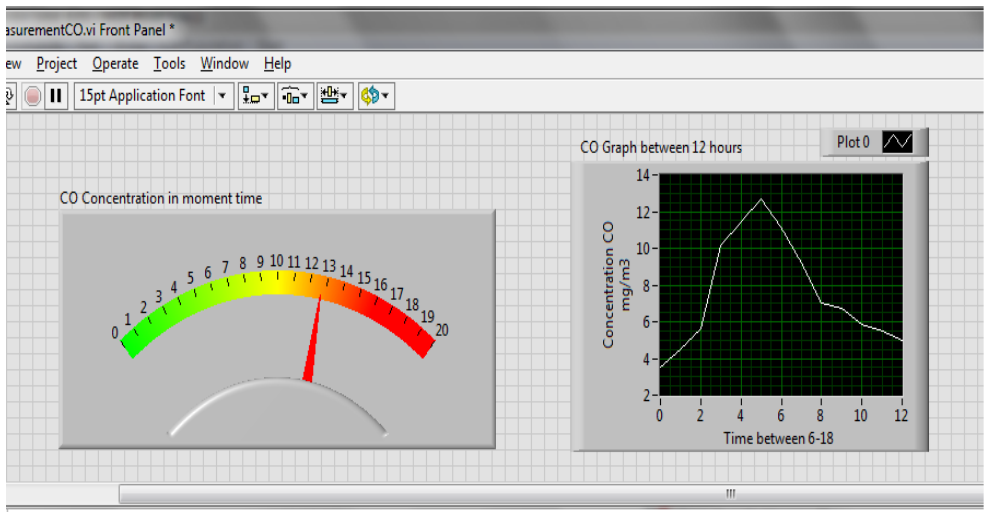

Fig. 6. The panel of virtual instrument that measures the concentration of carbon monoxide

Furthermore, the paper present the automatic acquisition, processing and analysis of environment data system, which is the analysis of the AHP multi-criteria method implemented within the system. The virtual instruments of our integrated system are based on objected-oriented programming. The input data is processed by using the LabVIEW functions. With the help of these functions the data is memorised in a database, in order to be further used in the realization of the objected classes for the generation of the intelligent maps of the Geomedia Professional Software.

The chemical factors which designed system can measure are: COx, PM10 dust and NOx.

The measurements were carried out in 4 different points from Sibiu: Union square, AlbaIulia street, DN $1306 \mathrm{~km}$ point and the Sub Arini park.

Virtual instrument design enables the implementation of multi-criteria analysis method works as follows:

- A subjective appreciation of the importance of each point stated above is made depending on the number of vehicles, number of people that are found in that concrete point at a 
certain time. The comparison matrix of pairs is realized, the weight of each observation point is calculated, the priority vector $\lambda$ (lambda), CR and CI are being calculated. The diagram of virtual instrument are presented in figure 7.

- Depending on the measurements made for each of the polluting factors, the virtual apparatus will calculate the weight of each polluting factor from the observation points taken into account.

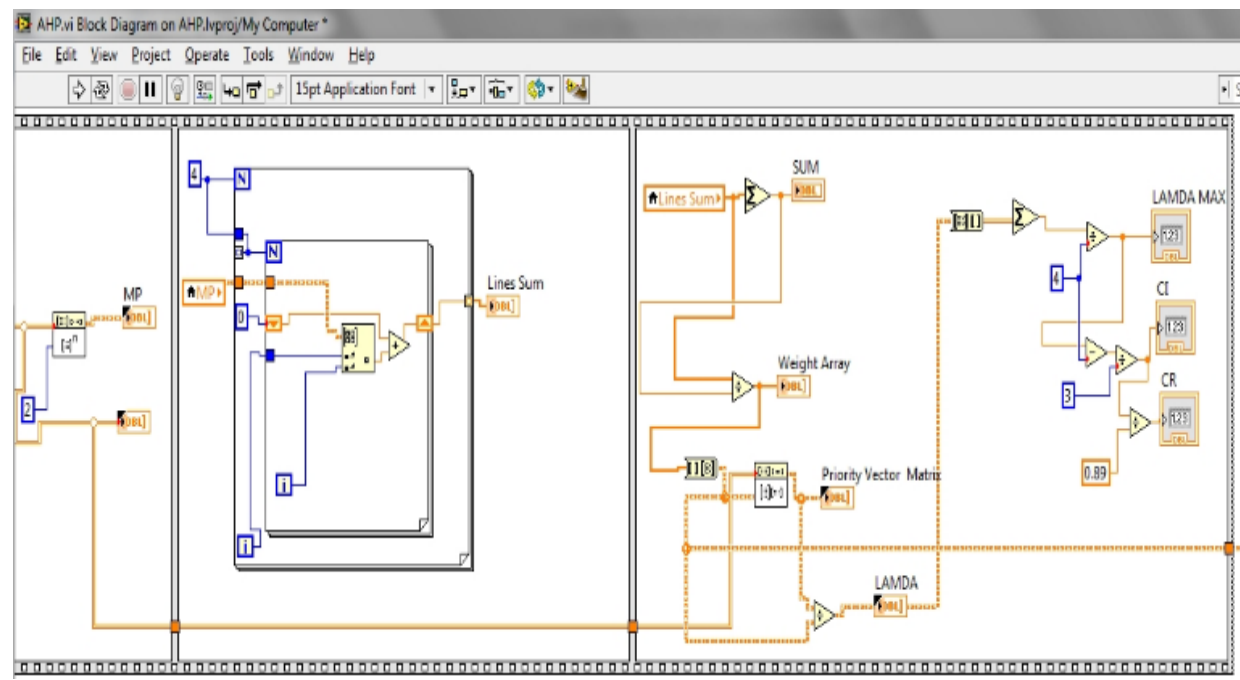

Fig 7. The diagram of VI for measurement points in AHP analysis

- Finally, the matrix of polluting factors is obtained. The matrix will be multiplied with the observation point's weights matrix. The virtual instrument allows the determination of the most and least polluted observation point, depending on the subjective appreciation made upon them. The final results are presented in the panel of virtual instrument created with Labview software, figure 8. Lambda and lambda max parameters to be calculated for obtaining CI and CR. Depending on their size choice was correct or not.

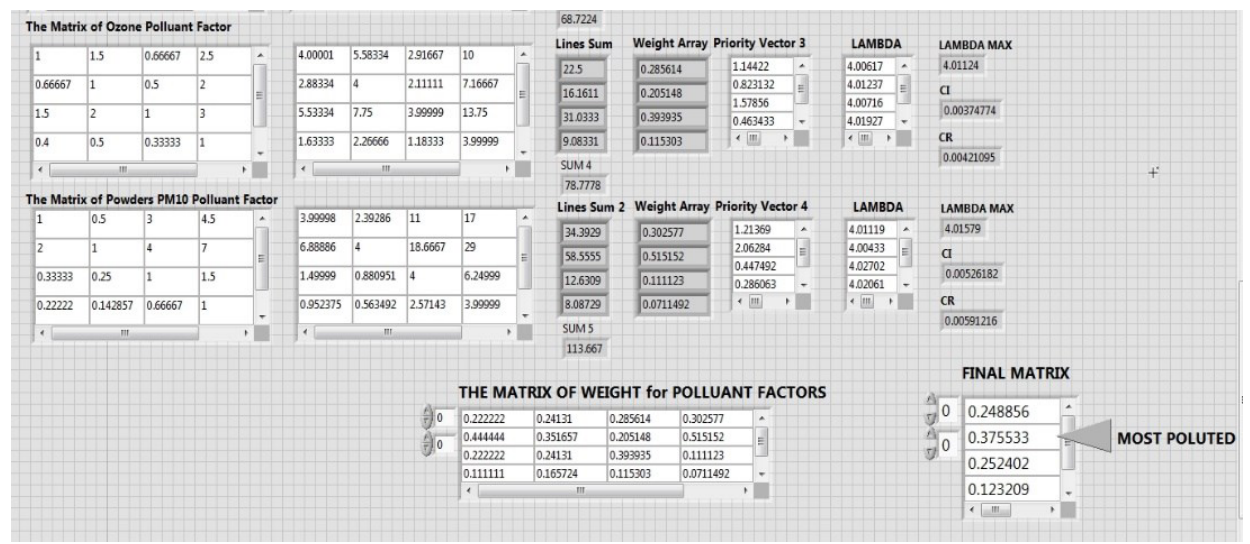

Fig. 8. The panel of virtual instrument with the matrix of weight for polluant factors and final matrix with results

The data is then retrieved from the Access database, in the software Geomedia Professional and intelligence map is generated by the polluted measuring point and the measurement point less polluted. For the examples presented, we can observe that the most 
polluted point is DN 1 at $306 \mathrm{~km}$, point, shopping area, followed by Alba Iulia street, Unirii Square and, as expected, Sub Arini Park, figure 9. The data taken with Labview software are stored in the database corresponding to virtual instrument. This Access database is then used by GIS software to generate proper intelligence map. On this map appear different colored points where measurements were made. Calculations using AHP multicriteria method allow to present in the map the most polluted and least polluted point, depending on chemical factors measured.

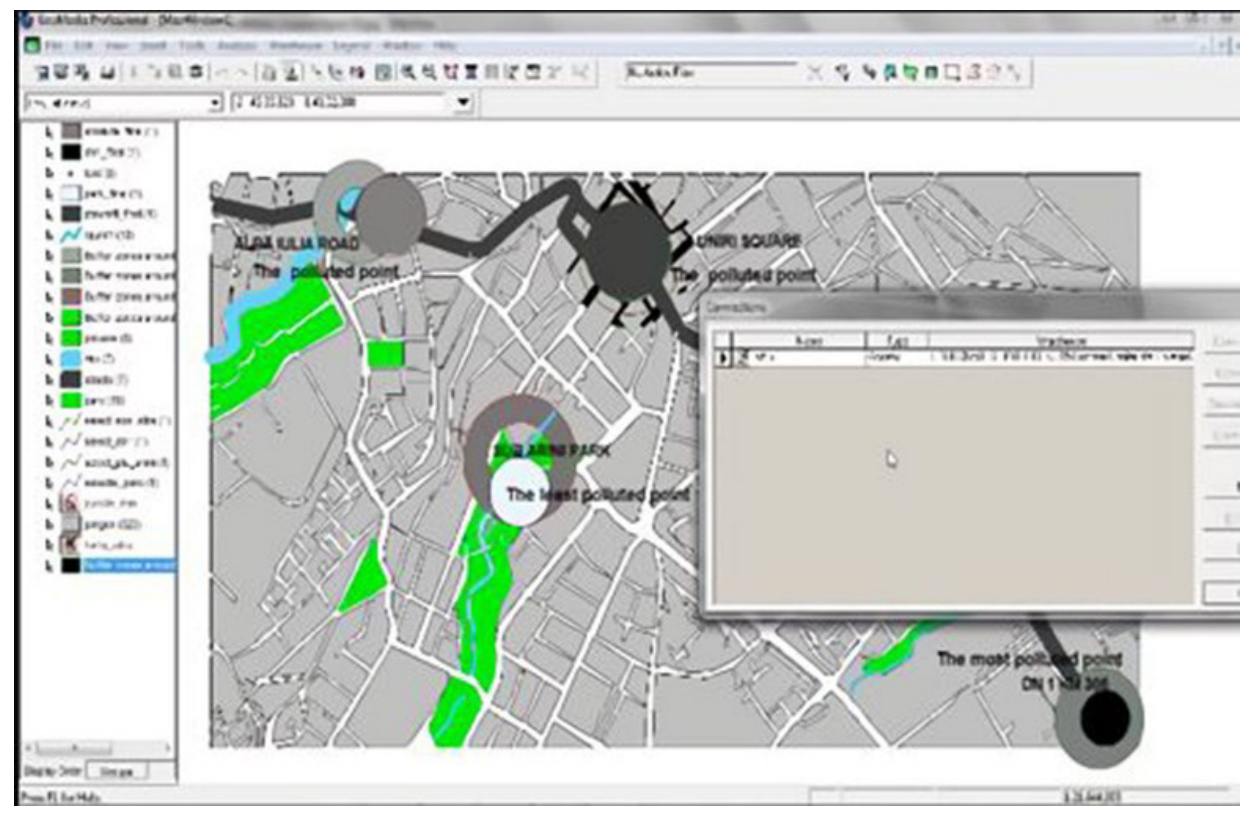

Fig. 9. The SIBIU map with the observation points and database connection

A very important thing to note is that the proposed automatic monitoring of environmental pollution factors system, allows obtaining environmental pollution situations in real time without human intervention. Data are obtained from the environment, is processed using virtual instrument, is stored in the common database with GIS software and then develops interactive map to view results.

In the future the system will be extended by using other MCDM analysis methods, like for instance the TOPSIS method. The system will be extended by implementing innovative methods based on genetic algorithms.

\section{References}

1. R. Mihaescu, Monitoringul Integrat al Mediului, (Cluj Napoca, 2014), http://enviro.ubbcluj.ro/studenti/cursuri\%20suport/Carte Monitoring_Radu SITE.pdf

2. O., Ciolpan, Monitoringul integrat al sistemelor ecologice, (Ars Docendi, București, 2005)

3. K. J. Mitchell, M. K., Johnson, M. Mather, Applied Cognitive Psychology Journal, 17(1), 1071119 (2003)

4. $* * *$ European Commission Communication from the Commission. Europe 2020. A strategy for smart, sustainable and inclusive growth. Brussels (2010).

5. C. Ioja, Metode de Cercetare si Evaluare a Starii Mediului (Ed. Etnologica, Bucuresti, 2013)

6. R. Bitter, T.Mohiuddin, M. Navrocki, LabView Advanced Programming Techniques (Boca Raton: CRC Press LLC, 2001)

7. D. Beck, H. Brand, Proceedings of ICALEPCS07 ( Knoxville, Tennessee, USA, 2014) 
8. J. Jehander, Graphical Object-Oriented Programming In LabVIEW - Tutorial - Developer Zone, National Instruments, http://labview360.com/document/an/pdf/an143.pdf, (2005)

9. T. L. Saaty, Int. J. Services Sciences, 1(1), 83-98 (2008)

10. B.G. Hermann, C. Kroeze, W. Jawjit, Journal of Cleaner Production 15 (18), 1787-1796 (2007)

11. Maliki, G. Owens, and D. Bruce, IPCBEE 37 (IACSIT Press, Singapore, 2012)

12. T. L. Saaty, The Analytic Hierarchy Process (McGraw-Hill, New York, 1980) 